\title{
DISEÑO SIMPLIFICADO DE SECCIONES METÁlICAS DE PLANCHA PLEGADA TIPO CAJÓN SUJETAS A FLEXO-COMPRESIÓN BIAXIAL
}

Francisco Aguirre Torrico y Álvaro Moscoso Wayar

\section{RESUMEN}

Este trabajo estudia el dimensionamiento simplificado de secciones metálicas de plancha plegada delgada tipo cajón sujetas a flexo compresión biaxial. Como no existen normas en nuestro país de estructuras metálicas, será utilizada como base la norma americana de diseño de estructuras metálicas de plancha plegada AISI S100 (2007). Se ha substituido la verificación del Unit Check tradicional de este tipo de solicitación por una expresión simple, transformando los momentos a una fuerza axial equivalente y junto con algunas tablas, el dimensionamiento es inmediato, sin necesidad de tentativas. Se han realizado verificaciones de este método de diseño simplificado y sus resultados se aproximan muy bien al del tradicional encontrado en la norma.

Palabras Clave: Estructuras Metálicas, Sección de Plancha Plegada, Flexo-Compresión Biaxial, Elementos VigaColumna. 